\title{
LA LIBRE CIRCULATION DES TRAVAILLEURS \\ ENTRE LA TURQUIE ET LA CEE
}

\section{LA LIBRE CIRCULATION DES TRAVAILLEURS ENTRE LA TURQUIE ET LA CEE(X)}

\author{
Yrd. Doç. Dr. Nurettin BlLtCI(X)
}

\section{A) INTRODUCTION}

1. L'accord créant une association entre la Turquie et la Communauté économique européenne (ci-après dénommée "accord d'Ankara") a été signé le 12 septembre 1963 à Ankara par la république de Turquie, d'une part, les Etats membres de la CEE et la Communauté, d'autre part (CEE, jo 1964, p. 3687), ${ }^{\text {. }}$

L'accord d'Ankara a pour objet, aux termes de l'article 2, de promouvoir le renforcement continu et équilibré des relations commerciales et économiques entre les parties. II comporte trois phase:

-une phase préparatoire permettant à la Turquie, aux termes de l'article 3, de renforcer son économie, avec l'aide de la Communauté (1964-1972);

-une phase transitoire consacrée, selon l'article 4 , à la mise en place progressive d'une union dounaière et au rapprochement des politiques économiques (1973-1995);

-une phase définitive qui, aux termes de l'article 5, est fondée sur l'union douanière et implique le renforcement de la coordination des politiques économiques.

Aux termes de l'aricle 28 , l'accord doit permettre à terme d'examiner la possibilité d'une adhésion de la Turquie à la Communauté.

2. En vue d'arrêter les conditions, modalités et rythmes de la réalisation de la phase transitoire, les parties contractantes ont signé, le 23 novembre 1970, à Bruxelles, un protocole additionnel (ci-après dénommée "protocole") (CEE, JO L293, p. 1).

3. Ces deux textes (l'accord d'Ankara et le protocole) constituent la principale base juridique des relations entre la Turquie et la Communauté européenne.

(X) Ce travail est exposé en tant qune thèse annexe à la thèse de doctorat soutenue à l'Universite Libre de Bruxelles (mai 1990).

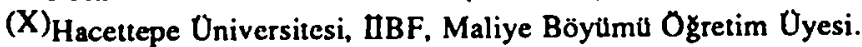

${ }^{1}$ Cet accord est enré en vigueur le ler décemre 1964. 
C'est en vertu de l'article 28 de l'accord d'Ankara que la Turquie a fait sa demande d'adhésion à la CEE en avril 1987.

\section{B) LES DONNEES RELATIVES A LA LIBRE CIRCULATION DES TRAVAILlEURS ENTRE LA CEE ET LA TURQUIE}

\section{a) Les textes}

4. L'accord d'Ankara et Le protocole contiennent chacun une disposition en ce qui conceme la libre circulation des travailleurs:

\section{- l'article 12 de l'accord d'Ankara dispose que}

"les parties contractantes conviennent de s'inspirer des articies 48, 49 et 50 du Traité ${ }^{2}$ instituant la Communauté pour réaliser graduellement la libre circulation des travailleurs entre elles."

L'article 36 de protocole dispose par ailleurs que:

"la libre circulation des travailleurs entre les Etats membres de la Communauté et la Turquie sera réalisée graduellement conformément aux principes énoncés à l'article 12 de l'Accord d'Association, entre la fin de la douziéme et de la vingt-deuxiéme année après l'entrée en vigueur dudit Accord.

\section{Le Conseil d'Association ${ }^{3}$ décidera des modalités nécessaires à cet effet.}

5. L'article 12 marque donc la volonté de réaliser par étapes, la libre circulation des travailleurs dans l'esprit des dispositions des articles 48 à 50 du traité CEE. C'est l'article 36 qui dit dans quelle durée sera réalisée ce libre circulation: c'est "...entre la fin de la douzième et de la vingt-deuxième année après l'entrée en vigueur dudit Accord." Par application de l'article 36 , la libre circulation de travailleurs devrait donc être réalisée dans la période allant de 30 novemre 1976 à 30 novembre 1986 étant donné que l'accord d'Ankara est entré en vigueur le ler décembre 1964.

b) L'arrêt du 30 septembre 1987 de la Cour de justice européenne ${ }^{4}$

aa) les faits

6. Selon une ressortissante turque (Mme Demirel), les dispositions de l'accord et du protocol devraient automatiquement donner droit à la libre circulation de travailleurs entre

\footnotetext{
${ }^{2}$ Ces articles règlementent la réalisation de la libre circulation des travailleurs entre les pays membres de la CEE.

${ }^{3}$ Le Conseil d'Association est composé, d'une part, de membres des gouvernements des Etats membres, du Conseil et de la Commission de la Communauté et, d'autre part, de membres du Gouvernement turc (l'accord, art. 22, 23). II statue à l'unanimité et dispose, pour la réalisation des objectifs fixés par l'accord, d'un pouvoir de décision.

${ }^{4}$ Affaire 12/86, en cause Demirel, Rec. p. 3719
} 


\section{LA LIBRE CIRCULATION DES TRAVAILLEURS ENTRE LA TURQUIE ET LA CEE}

la Turquie et la CEE. Elle a défendu cette thèse au cours d'un conflit déroulé entre elle et les autorités allemandes:

7. Elle était venue en Allemagne avec un visa délivré en Turquie pour rejoindre son marie. La durée de son visa expire et l'Administration allemande prend une décision d'expulsion a l'encontre d'elle. Mme demirel introduit ensuite un recours devant le Tribunal de Stuttgart tendant a l'annulation de cette décision d'expulsion. Dans son recours, elle fait référence aux articles 7,12 de l'accord a 36 de protocole. D'après elle, ces articles sont directement applicables dans l'ordre interne des Etats membres et instituent d'ores et déjà une interdiction dé droit de ces Etats établissant des restrictions à la libre circulation des turcs.

Le tribunal de Stuttgart considère que le litige comporte une appreciation de l'interprétation de l'accord d'association entre la CEE et la Turquie et décide en application de l'article 177 du traité CEE, de surseoir à statuer jusqu'à ce que la Cour de justice se soit prononcée à titre préjudiciel.

bb) La réponse de la Cour de justice européenne

8. La réponse de la Cour de justice européenne est donnée le 30 septembre 1987. Cette réponse est complètement décevante pour Mme Demirel. D'après la Cour,

lesdites textes (art. 12, 36) "revètent une portée essentiellement programmatique et ne constituent pas des dispositions suffisamment précises et inconditionnelles pour être susceptibles de régir directement la circulation des travailleurs.";

l'article 36 du protocole "attribue au seul conseil d'association compétence pour édicter des règles précises pour une réalisation graduelle de la libre circulation des travailleurs...";

"La seule décision que le conseil d'association a prise en la matière a été la décision $1 / 80$, du 19 septembre 1980, qui, à l'égard de travailleurs turcs déjà régulièrement intégrés au marche du travail des Etats membres, interdit toutes nouvelles restrictions en ce qui concerne les conditions d'accès à l'emploi."

9. L'arrêt souligne qu'une disposition d'un accord conclu par la Communauté avec des pays tiers ne doit être considérée comme étant d'application directe que:

"lorsque, eu égard à ses termes sinsi qu'à l'object et a la nature de l'accord, elle comporte une obligation claire et précise, qui n'est subordonnée, dons son exécution ou dans ses effets, l'intervention d'aucun acte ultérieur". 5

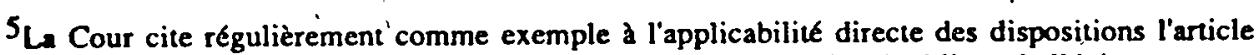
45. par. ler de "l'acte relatif aux conditions d'adhésion de la république hellénique et aux adaptations des traités" (JO (CEE), 1979, L 291, p. 17).

Cet article visait la libre circulation des travailleurs entre la CEE et la Grèce et aux termes duquel: 
En conséquence, dit l'arrêt qu'il y a lieu de répondre à la question du tribunal administratif de Stutgart que lesdites dispositions

"ne constituent pas des règles de droit communaitaire directement applicables dans l'ordre interne des Etats membres."

\section{C) CONCLUSION}

10. La Turquie et la CEE se sont mis d'accord en 1970 sur un calendrier afin de réaliser la libre circulation des travailleurs au cours d'une période allant de l'année 1975 à 1985. II est vrai que le fonctionnement de ce calendrier dépendait de l'initiative du Conseil d'association et, lequel n'a malheuresement rien fait dû au manque d'interêt du côté de la CEE.

Nous n'avons pas d'objection contre l'arrêt de la Cour de justice européenne. Notre objection est contre le Conseil d'Association qui n'a pas mis en oeuvre le calendrier prévu. Nous voulons simplement rappeler que l'article 7 de l'Accord d'Ankara "impose aux parties contractantes une obligation générale de coopération' en vue d'atteindre les objectifs de l'accord"6. Aux termes de cet article:

"les Parties contractantes prennent toutes lés mesures générales ou particulières propres à assurer l'exécution des obligations découlant de l'accord.

Elles s'abstiennent de toutes mesures susceptibles de mettre en péril la réalisation des buts de l'accord".

11. Rappelons qu'actuellement la Turquie insiste pour la réalisation des objectifs fixées par l'accord et notamment pour celle de la libre circulation des travailleurs. Par contre, de coté de la CEE, il n'y a pas d'autant d'intérêt. L'avis de la Commission ${ }^{7}$ note à ce propos:

"l'accés de la main-d'oeuvre turque au marché du travail de la Communauté qui devrait intervenir, même si ce n'est qu'au terme d'une période transitoire, suscite des appréhensions, en particulier tant niveau élevé de chomage persistera dans la Communauté."

L'existence du chomage dans la Communauté constitue l'objection principale de la CEE à la réalisation de libre circulation des travailleurs.

Cette objection est, à notre avis, privée de tout fondement juridique.

"les articles ...du règlement (CEE, 1968) relatif à la libre clrculation des travallleurs à l'intérleur de la Communauté ne sont appplicables ...à l'égard des ressortissants hellénlques ...qu'à partir du ler Janvier 1988 ."

${ }^{6}$ L'arrêt du 30 septembre 1987 , op. cit., p. 3754.

${ }^{7}$ L'avis de la Commission sur la Demande d'adhésion de la Turquiè à la Communauté. Commission des communautés européennes, Sec (89) 2290 final, Bruxelles, le 18 décembre 1989, p. 7, no:8.2. 